\title{
DUAL SERIES EQUATIONS INVOLVING GENERALIZED LAGUERRE POLYNOMIALS
}

B. M. SINGH, J. ROKNE, AND R. S. DHALIWAL

Received 1 April 2005

An exact solution is obtained for the dual series equations involving generalized Laguerre polynomials.

\section{Introduction}

We consider the following dual series equations:

$$
\begin{aligned}
& \sum_{n=0}^{\infty} \frac{A_{n} L_{n}^{(\alpha)}\left[(x+b)^{h}\right]}{\Gamma(\alpha+n+1)}=f(x), \quad 0<x<a, \\
& \sum_{n=0}^{\infty} \frac{A_{n} L_{n}^{(\sigma)}\left[(x+b)^{h}\right]}{\Gamma(\alpha+n+\beta)}=g(x), \quad a<x<\infty,
\end{aligned}
$$

where $\alpha+\beta+1>\beta>1-m, \sigma+1>\alpha+\beta>0, m$ is a positive integer, and $0<h<\infty$, $0 \leq b<\infty$, and $h$ and $b$ are finite constants. $L_{n}^{(\alpha)}\left[(x+b)^{h}\right]$ is a Laguerre polynomial, $A_{n}$ are unknown coefficients, and $f(x)$ and $g(x)$ are prescribed functions.

Srivastava $[5,6]$ has solved the following dual series equations:

$$
\begin{array}{ll}
\sum_{n=0}^{\infty} \frac{A_{n} L_{n}^{(\alpha)}(x)}{\Gamma(\alpha+n+1)}=f(x), & 0<x<a, \\
\sum_{n=0}^{\infty} \frac{A_{n} L_{n}^{(\sigma)}(x)}{\Gamma(\alpha+n+\beta)}=g(x), & a<x<\infty .
\end{array}
$$

The triple series equations (1.3) and (1.4) are a special case of the dual series equations (1.1) and (1.2) when

$$
h=1, \quad b=0 .
$$

Recently, Lowndes and Srivastava [3] have solved the triple series equations involving Laguerre polynomials. References for the solutions of dual and triple series equations 
involving Laguerre polynomials are given in [3]. Connected to this work, references and solutions for dual series equations are given by Sneddon [4].

The dual series equations (1.1) and (1.2) are new in the literature and have importance due to the closed-form solution. The results of this note are shown to be in agreement with those of Srivastava [5]. The analysis is purely formal and no justification had been given for the change of the order of integrations and summation.

\section{Some useful results}

In this section, we will discuss some results which are useful in solving dual series equations (1.1) and (1.2). The orthogonality relation for Laguerre polynomials is given by [2, page 292, equation (2)] and [2, page 293, equation (2)], from which we have

$$
\int_{0}^{\infty} x^{\alpha} e^{-x} L_{n}^{(\alpha)}(x) L_{m}^{(\alpha)}(x) d x=\frac{\Gamma(\alpha+n+1)}{\Gamma(n+1)} \delta_{n m}, \quad \alpha>-1,
$$

where $\delta_{n m}$ is the Kronecker delta.

We can easily find, with the help of integrals [2, page 293, equation (5)] and [2, page 405 , equation (20)], that

$$
\begin{aligned}
& \int_{0}^{\xi} x^{\alpha}(\xi-x)^{\beta+m-2} L_{n}^{(\alpha)}(x) d x \\
& \quad=\frac{\Gamma(\alpha+n+1) \Gamma(\beta+m-1)}{\Gamma(\alpha+\beta+m+n)} \xi^{\alpha+\beta+m-1} L_{n}^{(\alpha+\beta+m-1)}(\xi), \quad \alpha>-1, \beta+m>1, \\
& \int_{\xi}^{\infty} e^{-x}(x-\xi)^{\sigma-\alpha-\beta} L_{n}^{(\sigma)}(x) d x=\Gamma(\sigma-\alpha-\beta+1) e^{-\xi} L_{n}^{\alpha+\beta-1}(\xi), \quad \sigma+1>\alpha+\beta>0 .
\end{aligned}
$$

From [1, page 190, equation (27)], we find that

$$
\frac{d^{m}}{d x^{m}}\left[x^{\alpha+m} L_{n}^{(\alpha+m)}(x)\right]=\frac{\Gamma(\alpha+m+n+1)}{\Gamma(\alpha+n+1)} x^{\alpha} L_{n}^{\alpha}(x) .
$$

\section{Solution of dual series equations (1.1) and (1.2)}

We assume that

$$
\begin{gathered}
x+b=X^{1 / h}, \quad f\left(X^{1 / h}-b\right)=f_{1}(X), \\
g\left(X^{1 / h}-b\right)=g_{1}(X), \quad b^{h}=c, \quad(a+b)^{h}=d,
\end{gathered}
$$

then the dual series equations (1.1) and (1.2) can be written in the following form:

$$
\begin{array}{ll}
\sum_{n=0}^{\infty} \frac{A_{n} L_{n}^{(\alpha)}(X)}{\Gamma(\alpha+n+1)}=f_{1}(X), & c<X<d, \\
\sum_{n=0}^{\infty} \frac{A_{n} L_{n}^{(\sigma)}(X)}{\Gamma(\alpha+\beta+n)}=g_{1}(X), & d<X<\infty .
\end{array}
$$


We assume that

$$
\sum_{n=0}^{\infty} \frac{A_{n} L_{n}^{(\alpha)}(X)}{\Gamma(\alpha+n+1)}=f_{2}(X), \quad 0<X<c
$$

Combining the series equations (3.2) and (3.4), we can write the dual series equations (3.2) and (3.3) in the form

$$
\begin{array}{ll}
\sum_{n=0}^{\infty} \frac{A_{n} L_{n}^{(\alpha)}(X)}{\Gamma(\alpha+n+1)}=F(X), & 0<X<d, \\
\sum_{n=0}^{\infty} \frac{A_{n} L_{n}^{(\sigma)}(X)}{\Gamma(\alpha+\beta+n)}=g_{1}(X), & d<X<\infty,
\end{array}
$$

where

$$
F(X)= \begin{cases}f_{2}(X), & 0<X<c \\ f_{1}(X), & c<X<d .\end{cases}
$$

Multiplying (3.5) by $X^{\alpha}(\xi-X)^{\beta+m-2}$, where $m$ is a positive integer, integrating with respect to $X$ over $(0, \xi)$, and interchanging the order of integrations, we find on using (2.2) that

$$
\sum_{n=0}^{\infty} \frac{A_{n} L_{n}^{(\alpha+\beta+m-1)}(\xi)}{\Gamma(\alpha+\beta+m+n)}=\frac{\xi^{-\alpha-\beta-m+1}}{\Gamma(\beta+m-1)} \int_{0}^{\xi} X^{\alpha}(\xi-X)^{\beta+m-2} F(X) d X, \quad 0<\xi<d,
$$

where

$$
\alpha>-1, \quad \beta+m>1
$$

If we now multiply (3.8) by $\xi^{\alpha+\beta+m-1}$, differentiate both sides $m$ times with respect to $\xi$, and use formula (2.4), we find that

$$
\sum_{n=0}^{\infty} \frac{A_{n} L_{n}^{(\alpha+\beta-1)}(\xi)}{\Gamma(\alpha+\beta+n)}=\frac{\xi^{-\alpha-\beta+1}}{\Gamma(\beta+m-1)} \frac{d^{m}}{d \xi^{m}} \int_{0}^{\xi} X^{\alpha}(\xi-X)^{\beta+m-2} F(X) d X, \quad 0<\xi<d,
$$

where

$$
\alpha>-1, \quad \beta+m>1 .
$$


Multiplying (3.6) by $e^{-X}(X-\xi)^{\sigma-\alpha-\beta}$, integrating with respect to $x$ over $(\xi, \infty)$, and interchanging the order of integrations, we find by using formula (2.3) that

$$
\sum_{n=0}^{\infty} \frac{A_{n} L_{n}^{(\alpha+\beta-1)}(\xi)}{\Gamma(\alpha+\beta+n)}=\frac{e^{\xi}}{\Gamma(\sigma-\alpha-\beta+1)} \int_{\xi}^{\infty} e^{-X}(X-\xi)^{\sigma-\alpha-\beta} g_{1}(X) d X, \quad d<\xi<\infty,
$$

where

$$
\sigma+1>\alpha+\beta>0
$$

The left-hand sides of (3.10) and (3.12) are now identical. Making use of the orthogonality relation (2.1), we find from (3.10) and (3.12) that

$$
A_{n}=\Gamma(n+1)\left[\int_{0}^{d} \frac{e^{-\xi} L_{n}^{(\alpha+\beta-1)}(\xi) F_{1}(\xi) d \xi}{\Gamma(\beta+m-1)}+\int_{d}^{\infty} \frac{\xi^{\alpha+\beta-1} L_{n}^{(\alpha+\beta-1)}(\xi) G(\xi) d \xi}{\Gamma(\sigma-\alpha-\beta+1)}\right],
$$

where

$$
\begin{gathered}
F_{1}(\xi)=\frac{d^{m}}{d \xi^{m}} \int_{0}^{\xi} X^{\alpha}(\xi-X)^{\beta+m-2} F(X) d X, \\
G(X)=\int_{\xi}^{\infty} e^{-X}(X-\xi)^{\sigma-\alpha-\beta} g_{1}(X) d X
\end{gathered}
$$

provided that $\alpha+\beta+1>1-m$ and $\sigma+1>\alpha+\beta>0$.

With the help of (3.7), (3.15) can be written in the form:

$$
F_{1}(\xi)=\frac{d^{m}}{d \xi^{m}}\left[\int_{0}^{c} X^{\alpha}(\xi-X)^{\beta+m-2} f_{2}(X) d X+\int_{X}^{\xi} X^{\alpha}(\xi-X)^{\beta+m-2} f_{1}(X) d X\right], \quad c<\xi .
$$

When we put

$$
b=0, \quad h=1, \quad f_{2}(X)=0
$$

in the solution of the dual series equations (1.1) and (1.2), we then obtain the solution of the dual series equations (1.3) and (1.4) and the results are in complete agreement with those of [5].

\section{References}

[1] A. Erdélyi, W. Magnus, F. Oberhettinger, and F. G. Tricomi, Higher Transcendental Functions. Vols. I, II, McGraw-Hill, New York, 1953.

[2] , Tables of Integral Transforms. Vol. II, McGraw-Hill, New York, 1954.

[3] J. S. Lowndes and H. M. Srivastava, Some triple series and triple integral equations, J. Math. Anal. Appl. 150 (1990), no. 1, 181-187.

[4] I. N. Sneddon, Mixed Boundary Value Problems in Potential Theory, North-Holland, Amsterdam, 1966.

[5] H. M. Srivastava, A note on certain dual series equations involving Laguerre polynomials, Pacific J. Math. 30 (1969), 525-527. 
B. M. Singh et al. 1139

[6] _ Dual series relations involving generalized Laguerre polynomials, J. Math. Anal. Appl. 31 (1970), 587-594.

B. M. Singh: Department of Mathematics and Statistics, The University of Calgary, Calgary, AB, Canada T2N 1N4

J. Rokne: Department of Computer Science, The University of Calgary, Calgary, AB, Canada T2N $1 \mathrm{~N} 4$

E-mail address: rokne@cpsc.ucalgary.ca

R. S. Dhaliwal: Department of Mathematics and Statistics, The University of Calgary, Calgary, AB, Canada T2N 1N4

E-mail addresses: dhaliwal@math.ucalgary.ca; dhali.r@shaw.ca 


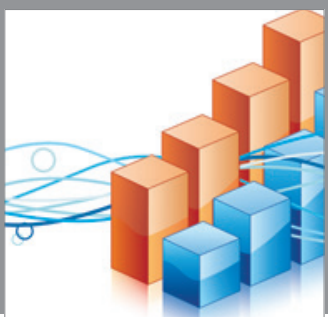

Advances in

Operations Research

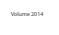

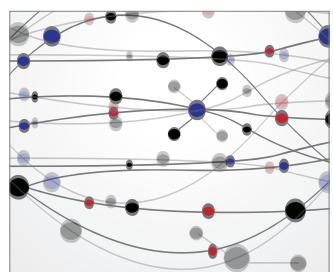

\section{The Scientific} World Journal
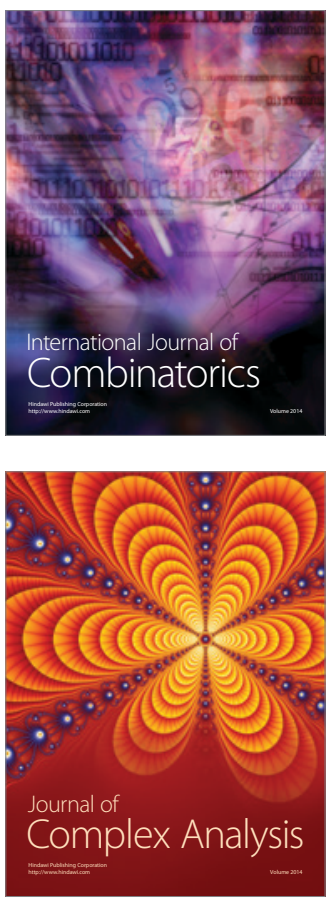

International Journal of

Mathematics and

Mathematical

Sciences
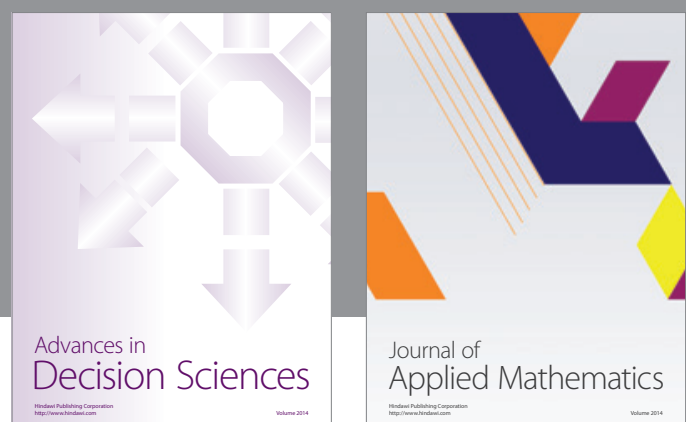

Journal of

Applied Mathematics
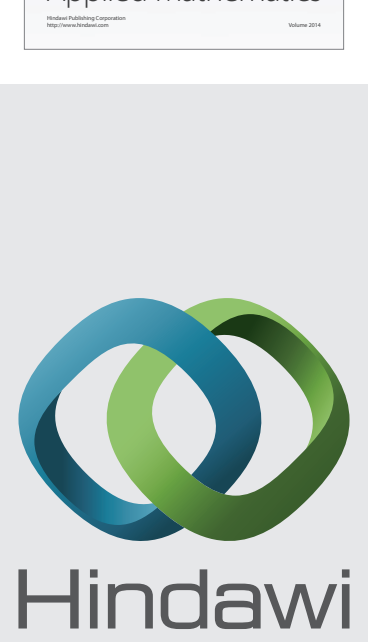

Submit your manuscripts at http://www.hindawi.com
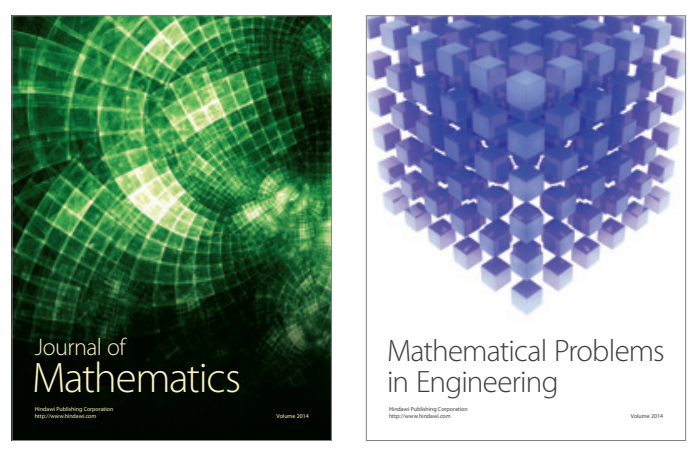

Mathematical Problems in Engineering
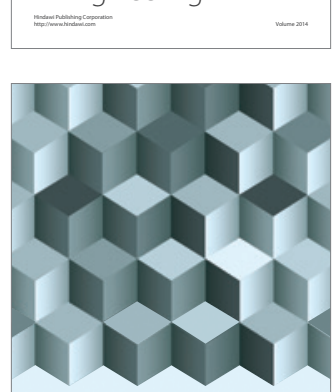

Journal of

Function Spaces
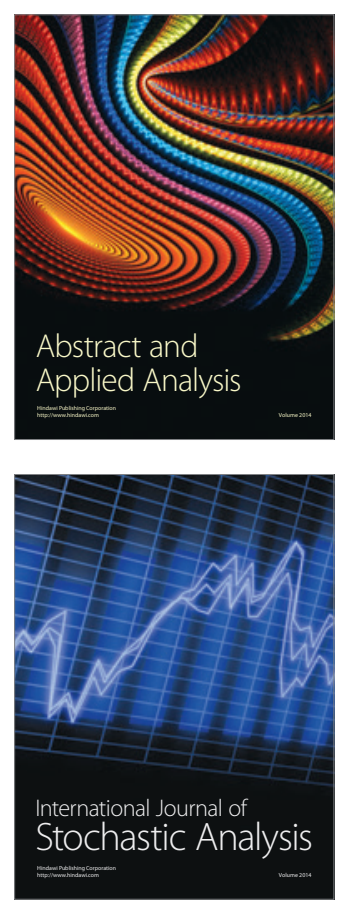

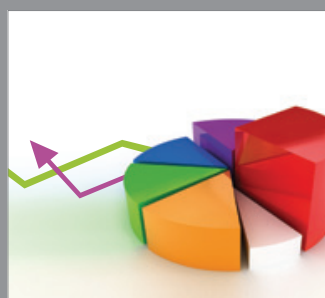

ournal of

Probability and Statistics

Promensencen
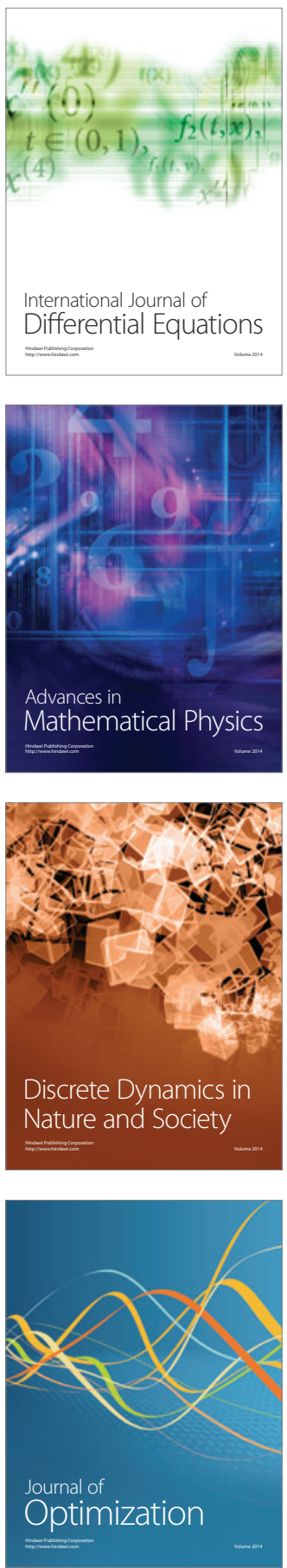